\title{
FDTD Analysis on Extraction Efficiency of GaN Light-Emitting Diodes With Microsphere Arrays
}

\author{
Peifen Zhu, Guangyu Liu, Jing Zhang, and Nelson Tansu
}

\begin{abstract}
The improvement of light extraction efficiency of InGaN light-emitting diodes (LEDs) using microsphere arrays with various refractive indices was analyzed. Finite-difference time-domain (FDTD) simulations show that the use of microsphere $\left(d_{\text {microsphere }}=500 \mathrm{~nm}\right)$ arrays with refractive indices of 1.8 and 2.5 led to increase in light extraction efficiency of InGaN LEDs by 1.9 times and 2.2 times, respectively. The enhancement in light extraction efficiency is attributed to the decrease in the Fresnel reflection and increase in effective photon escape cone due to graded refractive index and curvature formed between microsphere and free space. The maximum enhancement of light extraction efficiency of InGaN quantum well LEDs was achieved by employing the refractive index matched anatase- $\mathrm{TiO}_{2}$ microsphere arrays. The effects of microsphere diameters on the light extraction efficiency were also investigated and 2.4 times enhancement was achieved by employing $400-\mathrm{nm}$ refractive index matched $\mathrm{TiO}_{2}$ sphere arrays.
\end{abstract}

Index Terms - III-nitride, light-emitting diodes (LEDs), light extraction efficiency, matched refractive index, microspheres.

\section{INTRODUCTION}

$\mathbf{T}$ HE III-Nitride materials have an important use for addressing device technologies applicable in solid state lighting [1]-[8], thermoelectrics [9], [10], diode lasers [11], [12], and solar cells [13]. Among them, GaN based light-emitting diodes (LEDs) have drawn a lot of attention in recent years for a variety of applications in flat-panel displays, mobile electronics, automobiles, traffic signals, large outdoor displays, and general lighting. However, for the next generation of applications of high-efficiency LEDs, further improvement of external quantum efficiency of LEDs is required. The external quantum efficiency depends on the internal quantum efficiency and light extraction efficiency [14]. The advances in active region optimization and growth methods had led to improvement in internal quantum efficiency in recent years [15]-[19]. However, the light extraction efficiency of conventional LED is still low due to large refractive index contrast between $\mathrm{GaN}(\sim 2.5$ in the visible region) and free space. Therefore, various methods

Manuscript received January 18, 2013; revised February 14, 2013; accepted February 26, 2013. Date of publication March 11, 2013; date of current version March 26, 2013. The work was supported by U.S. Department of Energy (under Grant NETL DE-PS26-08NT00290, by the National Science Foundation under Grant ECCS 1028490 and Grant CBET 0828426, and by the Class of 1961 Professorship Fund.

The authors are with the Center for Photonics and Nanoelectronics, Department of Electrical and Computer Engineering, Lehigh University, Bethlehem, PA 18015 USA (e-mail: pez311@Lehigh.Edu; Tansu@Lehigh.Edu).

Color versions of one or more of the figures are available online at http:// ieeexplore.ieee.org.

Digital Object Identifier 10.1109/JDT.2013.2250253 have been employed to improve the light extraction efficiency of LEDs: 1) surface roughening [20]-[24]; 2) sapphire microlenses [25]; 3) oblique mesa sidewall [26]; 4) nanopyramid [27]; 5) photonic crystals [28]-[32]; 6) graded refractive index [33]; 7) self-assembled lithography p-GaN patterning [34]; 8) GaN micro-domes [35], [36]; and 9) $\mathrm{TiO}_{2}$ micro-pillars [37]. The surface roughening approach utilizes chemical etching, which cause non-uniform surface. The photonic crystals and sapphire microlens approaches require the use of e-beam lithography or holography lithography [25], which leads to more expensive and/or complex fabrication process. Therefore, other low-cost and large area scalable method is highly desirable for implementation for low-cost and practical LEDs technologies.

Previously, we have demonstrated the use of $\mathrm{SiO}_{2}$ microsphere arrays and $\mathrm{SiO}_{2} / \mathrm{PS}$ microlens arrays, deposited via rapid convective deposition (RCD) method, led to improvement in light extraction efficiency in III-Nitride LEDs [38]-[41]. The use of RCD method [38], [40] led to the ability for deposition of large area (wafer scale) microsphere (and/or microlens) arrays structures on top of LED device structures. Recently, this colloidal lithography method had been used as imprinting template for forming concave microstructure arrays for light extraction enhancement in GaN-based LEDs [42] and organic-based LEDs [43]. The selection of $\mathrm{SiO}_{2}$ as the microspheres in our previous works [38]-[41] had been primarily motivated from the ease in obtaining the silica-based spheres with wide range of dimensions commercially. However, the material choices (i.e., refractive indices) and dimensions of the spheres are design parameters that need to be optimized for maximizing the light extraction efficiency of nitride-based LEDs. In addition, the selection of spheres with various refractive indices and dimensions will also affect the far-field radiation pattern of the LEDs over wide angular distribution.

In this work, we present the numerical simulation studies based on finite-difference time-domain (FDTD) method for analyzing the light extraction efficiency of III-Nitride LEDs employing microsphere arrays with various refractive indices and dimensions. Specifically, the light extraction characteristics of III-Nitride LEDs with anatase- $\mathrm{TiO}_{2}$ and amorphous- $\mathrm{TiO}_{2}$ microsphere arrays were compared with those of III-Nitride LEDs with $\mathrm{SiO}_{2}$ microsphere arrays. The refractive indices of anatase- $\mathrm{TiO}_{2}$ and amorphous- $\mathrm{TiO}_{2}$ microsphere arrays are higher than that of $\mathrm{SiO}_{2}$ microsphere, which will lead to the improved extraction characteristics for III-Nitride LEDs employing the $\mathrm{TiO}_{2}$ microsphere arrays. The comparison of the light extraction efficiency enhancement for LEDs employing lower refractive index sphere $\mathrm{SiO}_{2}$, medium refractive index sphere amorphous- $\mathrm{TiO}_{2}$, and higher refractive index sphere 


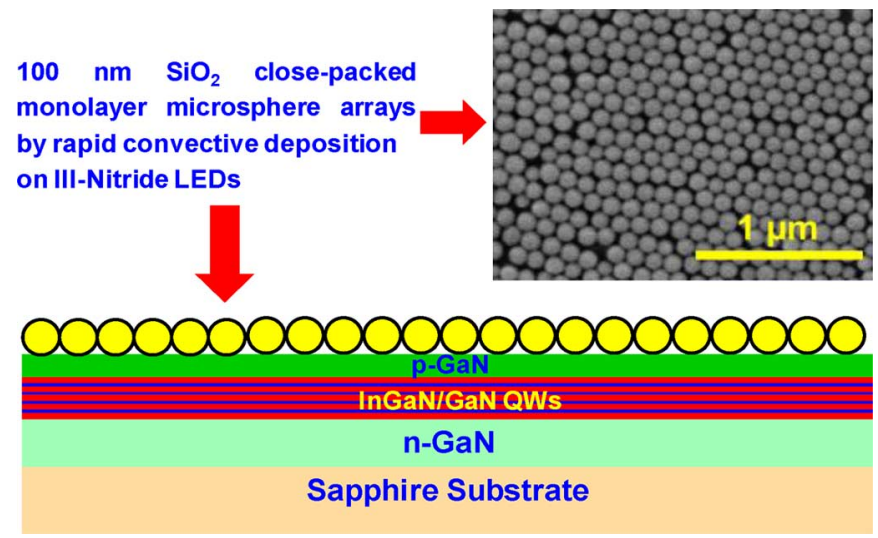

Fig. 1. Schematic side view of the simulated microsphere LED device, and the corresponding SEM images of $100-\mathrm{nm}-\mathrm{SiO}_{2}$ sphere arrays.

anatase- $\mathrm{TiO}_{2}$ with various diameters will be presented. In contrast of the microsphere arrays LEDs, the fabrication of the microlens arrays can be formed by embedding the microsphere arrays with polystyrene (PS) materials (i.e., $\mathrm{SiO}_{2} / \mathrm{PS}$ microlens arrays [38]-[41]). However, the current study will only be limited to the light extraction efficiency optimization in GaN-based LEDs employing only microsphere arrays with various materials, refractive indices, and diameters.

\section{FDTD ANALYSIS OF Light EXTRACTION EFFICIENCY}

The InGaN/GaN multiple quantum wells (MQWs) LED device structure analyzed in this study is shown in Fig. 1. The $\mathrm{n}-\mathrm{GaN}$ template was grown on sapphire substrate and followed by the growth of active region with total thickness of $12 \mathrm{~nm}$. Then $0.1 \mu \mathrm{m}$ p-doped GaN layer was grown on top. Afterwards, the hexagonal close-packed (HCP) microsphere arrays were deposited on top of LED by employing rapid convective deposition (RCD) method [38]-[42]. The deposition of the microspheres or nanospheres can be performed with high uniformity in close-packed 2-D hexagonal pattern, and the scanning electron microscopy (SEM) of the 100-nm diameter nanosphere arrays was shown in Fig. 1. Our prior works had demonstrated the deposition of micro/nanosphere arrays with diameters ranging from $100-\mathrm{nm}$ up to $1-\mu \mathrm{m}$ performed consistently on the devices [38]-[43].

The LED devices were treated as 3-D structures solved by taking into consideration the appropriate boundary conditions for ensuring efficient computation time. The 3-D FDTD method requires a large amount of memory and computation time. Therefore, the size of the simulation volume has to be reduced. Perfect matched layers (PML) boundary conditions were applied on the boundaries in order to avoid unnecessary reflection of light at the boundaries of domain as well as reduce the domain size. To reduce the size of the calculation, we have taken the simulation domain to be $5.5 \mu \mathrm{m} \times 5.5 \mu \mathrm{m}$. The thickness of $\mathrm{n}-\mathrm{GaN}$ reduced to $0.1 \mu \mathrm{m}$ and sapphire substrate was reduced to $0.2 \mu \mathrm{m}$.

A single dipole source within a finite computational domain was chosen and positioned in the center of $\mathrm{x}-\mathrm{y}$ plane and also in the center of the InGaN/GaN active layer in the vertical direction. In addition, inhomogeneous mesh was used during the

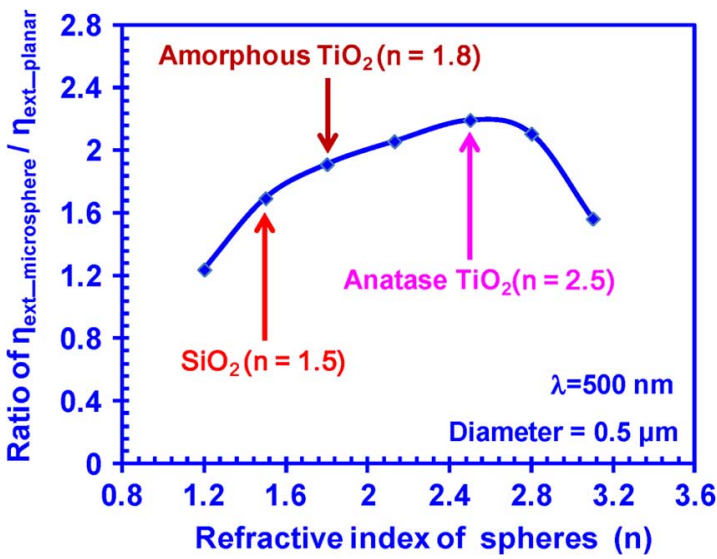

Fig. 2. Ratio of light extraction efficiency of microsphere array LEDs with various refractive indices to that of planar LED.

simulation; the grid size was $10 \mathrm{~nm}$ in the bulk and $2.4 \mathrm{~nm}$ in the interface. The refractive index of $\mathrm{GaN}$ and $\mathrm{InGaN}$ layer is set as 2.5 and 2.6, respectively. The absorption coefficient of InGaN was chosen to be $2000 \mathrm{~cm}^{-1}$ [15]. The emission wavelength used in all our computation is set as $\lambda=500 \mathrm{~nm}$. The light extraction efficiency was calculated as the ratio of the optical output power radiated through the microlens array to the total output power generated in the InGaN/GaN MQWs active region. Two detection monitors were implemented for measuring the electric field radiated from the dipole and near-field electric field on the top surface of the LEDs. The first monitor was formed to surround the dipole, in order to measure the total output power generated in the QW active region. The second monitor was placed $200-300 \mathrm{~nm}$ away from the top surface of the LEDs for measuring the near field electric field $\left[\vec{E}\left(x, y, z_{\text {monitor }}\right)\right]$ radiated from the device. The far-field electric field $[\vec{E}(r, \theta, \phi)]$ was obtained by performing the Fourier Transform on the near-field electric field $\left[\vec{E}\left(x, y, z_{\text {monitor }}\right)\right]$. The light extraction efficiency for LEDs is calculated by taking the ratio of the total power extracted from the LEDs, which was obtained by integrating the far-field power density over all solid angle, with the total power radiated the dipole as measured at the first monitor.

\section{EFFECT OF REFRACTIVE INDICES ON Light EXTRACTION EFFICIENCY OF MICROSPHERE ARRAY LEDS}

To investigate the effect of the refractive index of microsphere arrays on the light extraction efficiency of III-nitride LEDs, light extraction efficiency of GaN LEDs with microsphere $\left(\mathrm{d}_{\text {microsphere }}=500 \mathrm{~nm}\right)$ arrays and planar LED were computed for various refractive indices. The ratios of the light extraction efficiencies of the LEDs with microsphere arrays to those of the planar LED are shown in Fig. 2. Light extraction efficiency increases with the increase in refractive index of microsphere, and $\sim 2.2$ times enhancement was achieved when the refractive index of microsphere matched with that of $\mathrm{GaN}$. The enhancement started to decrease with further increase in the refractive index of the microspheres, which is expected to the increased light trapping in microspheres (for $\mathrm{n}>2.5$ ). To compare suitable microsphere materials applicable for the experi- 

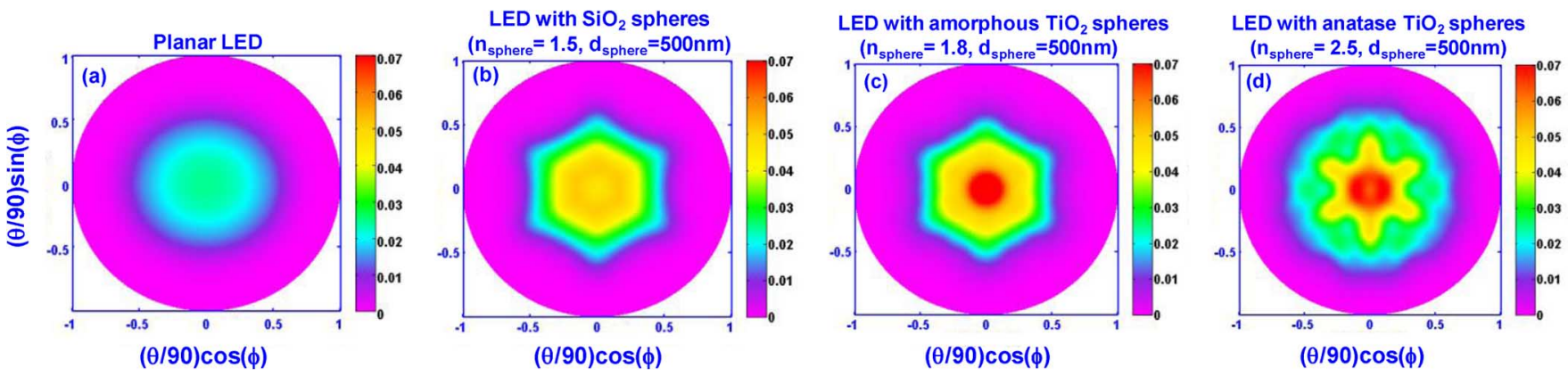

$(\theta / 90) \cos (\phi)$

Fig. 3. Contour plot of far-field radiations of (a) planar LED, (b) LED with $\mathrm{SiO}_{2}$ microsphere arrays, (c) LED with amorphous $\mathrm{TiO}_{2}$ microsphere arrays, and (d) LED with anatase $\mathrm{TiO}_{2}$ microsphere arrays.

ments, the following microspheres were investigated: 1) $\mathrm{SiO}_{2}$ $(\mathrm{n} \sim 1.5), 2)$ amorphous $\mathrm{TiO}_{2}(\mathrm{n} \sim 1.8)$, and 3$)$ anatase $\mathrm{TiO}_{2}(\mathrm{n} \sim 2.5)$. The computational analysis indicated that the use of $\mathrm{SiO}_{2}$ microsphere arrays $\left(\mathrm{d}_{\mathrm{SiO} 2}=500 \mathrm{~nm}\right)$ led to an increase of 1.7-times in the light extraction efficiency of the devices, which was in good agreement with the experiments ( 1.69 times increase) [39] and the simulation results employing ray tracing method (1.75 times enhancement) [39]. Note that simulation results using FDTD method is much closer to the experimental results compared to that of using ray tracing method. By using amorphous and anatase $\mathrm{TiO}_{2}$ microsphere arrays, the light extraction efficiencies of the LEDs increase by1.91-times and 2.19-times over that of planar LEDs. As the microsphere refractive indices increases beyond $\mathrm{n}>2.5$, the light trapping in the microspheres leads to reduction in the light extraction to free space.

The increase in the refractive indices of the microspheres up to $\mathrm{n} \sim 2.5$ provides minimal Fresnel reflection (between the $\mathrm{GaN}$ and spheres) resulting in efficient light capture in the spheres, which will then be extracted out efficiently by the increased escape cone from the strong curvature surfaces introduced by microsphere arrays.

As comparison, $\mathrm{SiO}_{2}$ film was deposited on the LEDs as antireflection coatings to reduce the Fresnel reflection and increase the light extraction efficiency. In the planar $\mathrm{SiO}_{2}$ antireflection coating film, the Fresnel reflection within the light escape cone will be significantly reduced. However, the use of $\mathrm{SiO}_{2}$ planar antireflection film will not increase the critical angle of the structure $\left(\theta_{\text {critical }}=23.5^{\circ}\right)$, of which is determined by the index contrasts of the GaN material and air (free space). Thus, the use of $\mathrm{SiO}_{2}$ antireflection thin film will not increase the light escape cone, which results in only reduced Fresnel reflection within the existing escape cone $\left(\theta_{\text {critical }}=23.5^{\circ}\right)$. In contrast, the role of the microsphere or nanosphere arrays is to provide strong scattering centers to extract light out beyond the critical angle, which in turn results in the increase in light extraction efficiency for the microsphere LEDs over LEDs with only planar $\mathrm{SiO}_{2}$ anti-reflecting film.

The comparison for the far-field radiation patterns for the planar LEDs, LEDs with $\mathrm{SiO}_{2}$ microspheres, LEDs with amorphous $\mathrm{TiO}_{2}$ microspheres, and LEDs with anatase $\mathrm{TiO}_{2}$ microspheres was presented in Figs. 3 and $4\left(d_{\text {microsphere }}=500 \mathrm{~nm}\right)$. The far-field radiation pattern for planar LEDs, as expected, ex-

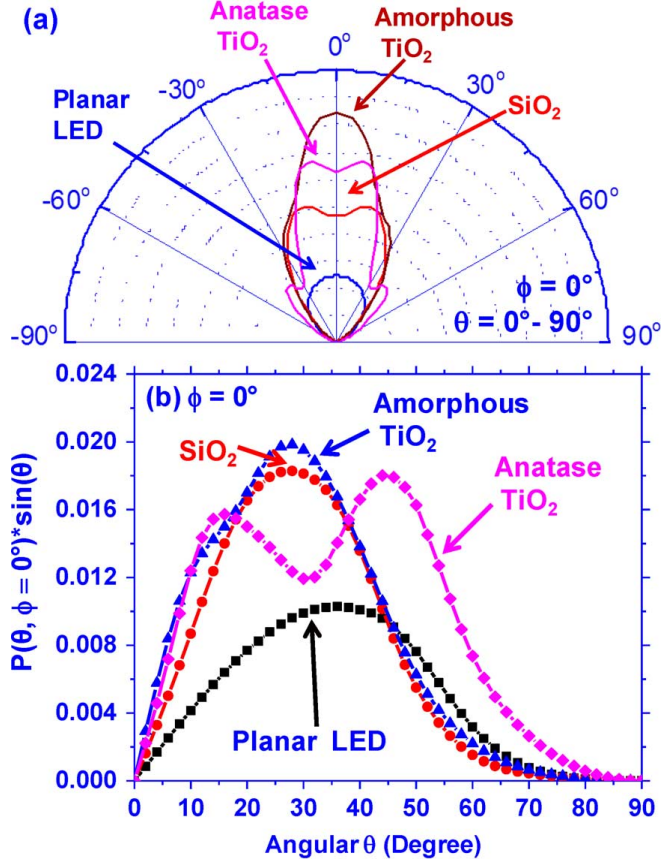

Fig. 4. (a) Polar plot of far-field intensities of microsphere LEDs with various refractive indices, and (b) angular dependent power density of microsphere LEDs with various refractive indices.

hibited Lambertian radiation pattern with only angular $(\theta)$ dependent, while the symmetrically azimuthal $(\phi)$ distribution. Note that the far-field radiation pattern for 2-D hexagonal closepacked microsphere arrays LEDs exhibits both angular $(\theta)$ and azimuthal $(\phi)$ dependent, as shown in Fig. 3(b)-(d). The significantly higher intensity was also observed for far-field radiation pattern for microsphere LEDs for both the normal and large angular distribution, which in turn results in improved light extraction efficiency for these LEDs. Note that the comparison of the light extraction efficiency ratio for the microsphere LEDs and planar LEDs were carried out by taking the total output power integrated in all angular $(\theta)$ and azimuthal $(\phi)$ directions.

In order to provide a quantitative comparison, the far-field radiation patterns for these LEDs were plotted at a particular azimuthal direction of $\phi=0$ [as shown in Fig. 4(a)]. The angular dependent (in $\theta$ direction) power density comparison data for microsphere LEDs and planar LED were also plotted for 
comparison purpose [as shown in Fig. 4(b)]. The microsphere LEDs exhibited significant increase in the far-field radiation patterns for all angular distribution. More importantly, the contribution of the large angular $\theta$ component in the far-field radiation pattern is significantly increased for anatase $\mathrm{TiO}_{2}$ microsphere arrays LEDs, which resulted in increase in power density extracted from this microsphere LEDs.

Note that the choice of the refractive indices of the materials $\left(\mathrm{SiO}_{2}\right.$, amorphous $\mathrm{TiO}_{2}$, and anatase $\left.\mathrm{TiO}_{2}\right)$ strongly affects the radiation pattern, extraction value, and power density profile at large angular direction. The studies presented in Figs. 3 and 4 assume identical diameters for all the microspheres investigated. However, both the refractive indices and diameters of the spheres are important as optimization parameters in LEDs extraction.

\section{EFFect of Microsphere Diameters on Light EXTRACTION OF MICROSPHERE LEDS}

In order to further optimize the light extraction efficiency of LEDs with microsphere arrays, the effect of diameter of spheres on the light extraction efficiency of microsphere LEDs was also investigated [as shown in Fig. 5(a), (b), and (c)]. Fig. 5(a) shows the light extraction efficiency enhancement of LEDs with $\mathrm{SiO}_{2}$ $(\mathrm{n} \sim 1.5)$ microsphere arrays as a function of diameters of spheres at $\lambda=500 \mathrm{~nm}$. All the three microsphere LEDs exhibited strong dependence on the diameter for the microsphere arrays employed in the structures. The optimum dimensions for each type of sphere materials occur at different dimensions resulting in maximum allowed enhancement in the microsphere LEDs. The comparison of the light extraction efficiencies for microsphere LEDs were carried out with that of planar LEDs serving as reference LEDs.

For the case of $\mathrm{SiO}_{2}$-based microsphere LEDs, the maximum extraction enhancement of 1.85 times is expected for diameter of $\sim 1 \mu \mathrm{m}$. Large range of $\mathrm{SiO}_{2}$ microsphere diameter exists for achieving extraction efficiency enhancement by $\sim 1.6$ times or higher. Specifically, the diameters in the range of $0.4 \mu \mathrm{m}$ up to $1.2 \mu \mathrm{m}$ are expected to result in relatively favorable increase in light extraction in LEDs.

For the case of amorphous $\mathrm{TiO}_{2}$ microsphere LEDs, the increase up to 2.1 times is observed for LEDs using optimum diameter $\mathrm{d} \sim 0.8 \mu \mathrm{m}$. The use of amorphous $\mathrm{TiO}_{2}$ microspheres with diameters ranging from $0.4 \mu \mathrm{m}$ up to $0.9 \mu \mathrm{m}$ is expected to result in increased extraction efficiency by 1.9 times or higher.

By using the anatase $\mathrm{TiO}_{2}$ microsphere arrays, the optimum diameter for this structure was obtained as $\mathrm{d} \sim 0.4 \mu \mathrm{m}$. The use of this optimum anatase $\mathrm{TiO}_{2}$ microsphere arrays with $\mathrm{d} \sim$ $0.4 \mu \mathrm{m}$ results in 2.4 times increase in light extraction efficiency over that of planar LEDs.

From our finding, the optimum diameter range for high extraction efficiency for each type of microsphere arrays reduces, as the refractive indices increases to $\mathrm{n} \sim 2.5\left(\mathrm{n}_{\mathrm{GaN}}\right)$. However, the maximum light extraction enhancement for the LEDs can be obtained from the microspheres with the refractive index closest to $\mathrm{n} \sim 2.5$ (anatase $\mathrm{TiO}_{2}$ ).

The comparison of the far-field radiation patterns of the $\mathrm{SiO}_{2}$ and anatase $\mathrm{TiO}_{2}$ (as optimized material) microsphere arrays
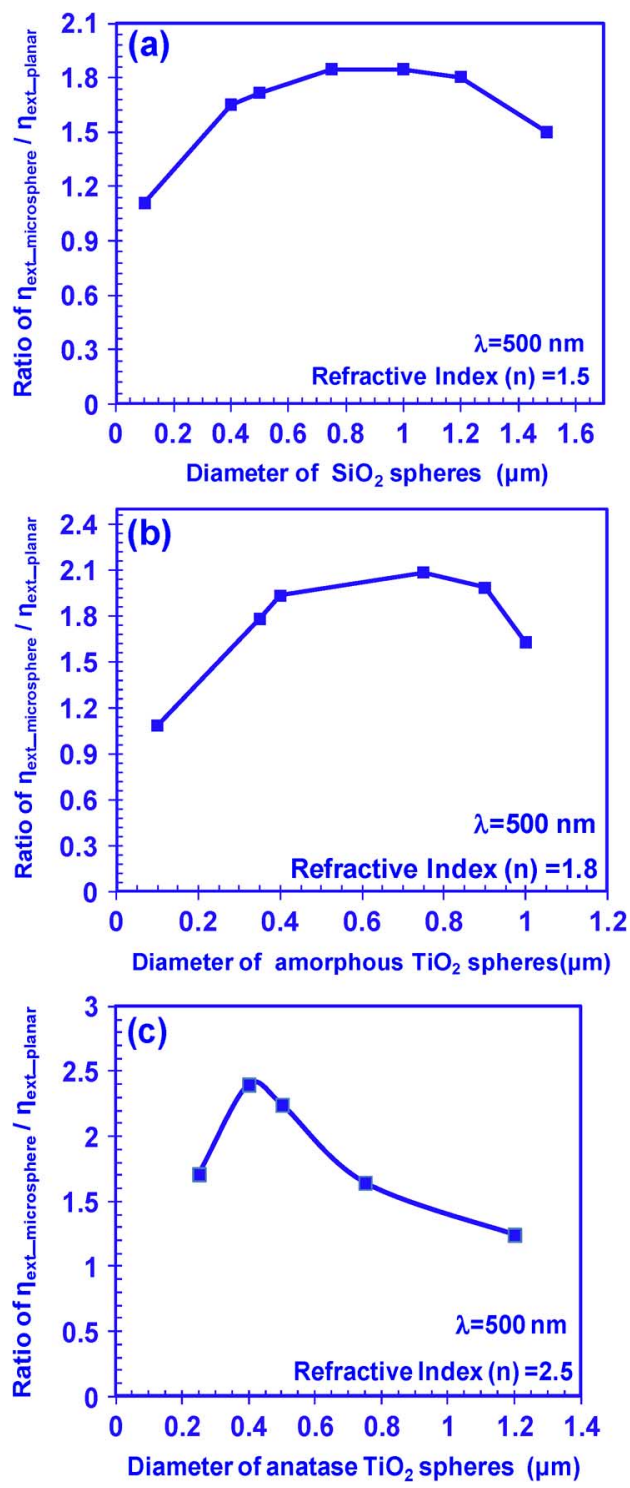

Fig. 5. Ratio of the light extraction efficiency of microsphere LEDs with various diameters to that of planar LED: (a) LEDs with $\mathrm{SiO}_{2}$ microsphere arrays; (b) LEDs with amorphous $\mathrm{TiO}_{2}$ microsphere arrays and (c) LEDs with anatase $\mathrm{TiO}_{2}$ microsphere arrays.

for various diameters were shown in Figs. 6 and 7. The comparison of the far-field patterns for both $\mathrm{SiO}_{2}$ [Fig. 6(a)] and $\mathrm{TiO}_{2}$ [Fig. 7(a)] microspheres were taken with azimuthal $\phi=0$ direction, in order to provide quantitative comparison between the two materials with various diameters. The comparison of the angular power density distributions for both $\mathrm{SiO}_{2}$ and $\mathrm{TiO}_{2}$ microsphere LEDs for various diameters were also shown in Figs. 6(b) and 7(b), respectively (with azimuthal $\phi=0$ direction).

In the case of $\mathrm{SiO}_{2}$ microsphere LEDs [Fig. 6(a) and (b)], the microsphere diameters were varied from $0.1 \mu \mathrm{m}, 0.4 \mu \mathrm{m}$, $0.5 \mu \mathrm{m}, 0.75 \mu \mathrm{m}$ and $1 \mu \mathrm{m}$. The use of small $\mathrm{SiO}_{2}$ microsphere $\left(\mathrm{d}_{\mathrm{SiO} 2}<0.1 \mu \mathrm{m}\right)$ leads to enhancement of light extraction at large angular component, while LEDs with larger sphere arrays $\left(\mathrm{d}_{\mathrm{SiO} 2}>0.5 \mu \mathrm{m}\right)$ results in significant increase in the output power in the normal direction. The large contribution of diffuse light output power can be attributed to the increased photon escape cone in the structure. 


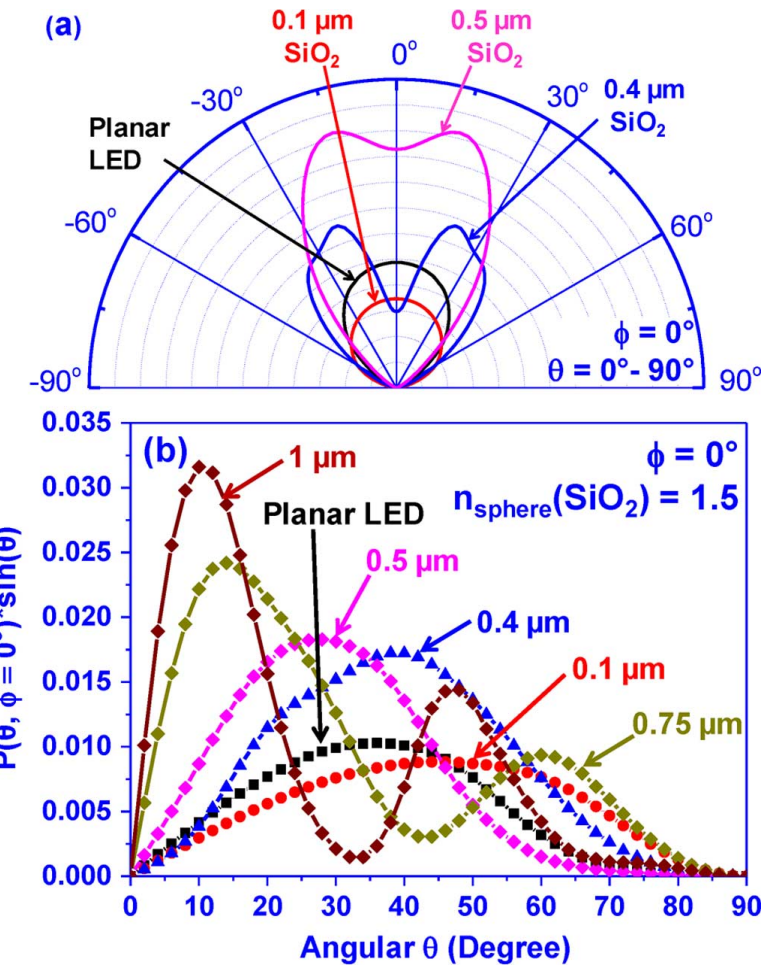

Fig. 6. (a) Far-field radiation patterns of $\mathrm{SiO}_{2}$ microsphere array LEDs with various $\mathrm{SiO}_{2}$ sphere diameters. (b) Angular dependent power density comparison of $\mathrm{SiO}_{2}$ microsphere array LEDs. The planar LED is included as reference.

In the analysis of the anatase- $\mathrm{TiO}_{2}$ microsphere array LEDs [Fig. 7(a) and (b)], the diameters were varied from $0.25 \mu \mathrm{m}$, $0.4 \mu \mathrm{m}$, and $0.75 \mu \mathrm{m}$. As shown in Fig. 7(b), the far-field radiation pattern for the anatase $\mathrm{TiO}_{2}$ microsphere LEDs were strongly enhanced in comparison to that of planar LEDs. Similar to the $\mathrm{SiO}_{2}$ microsphere LEDs, the use of smaller anatase $\mathrm{TiO}_{2}$ spheres results in strong enhancement in the large angular emission. In contrast, the dominant component leading to enhancement in extraction efficiency for larger anatase $\mathrm{TiO}_{2}$ spheres can be attributed to the stronger normal light emission.

\section{CONCLUSION}

The effects of refractive indices, material choices, and sphere diameters were investigated for optimizing the far-field radiation patterns and light extraction in III-Nitride based LEDs with microsphere arrays. The analysis was carried out by using 3-D vectorial FDTD with PML boundary condition. The results show that the use of microspheres with high refractive index as favorably for achieving maximum light extraction efficiency in microsphere LEDs. Specifically, the use of anatase $\mathrm{TiO}_{2}$ microsphere arrays is expected to result in more than 2.4 times increase in light extraction efficiency over that of planar LEDs. The optimum diameters of the spheres are found to strongly affect the radiation patterns and light extraction efficiency in microsphere LEDs. The use of smaller spheres results in significant enhancement in light extraction at large angular component, while the larger sphere appears to result in stronger enhancement in normal light emission. The current work is limited to the optimization of LEDs with microsphere arrays, and this (a)
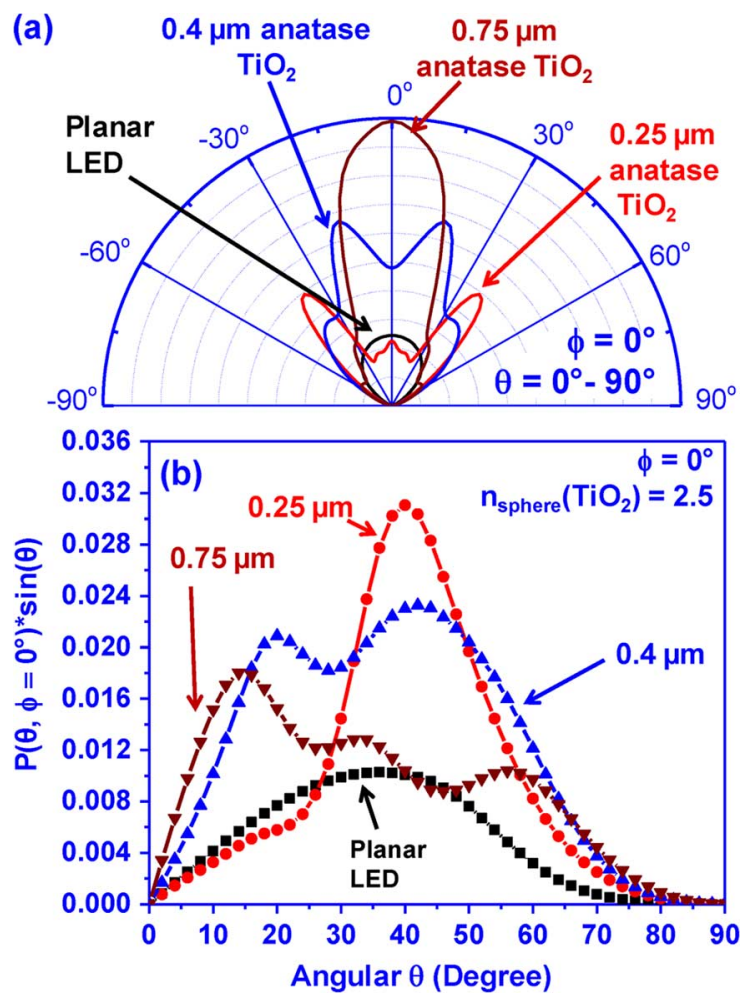

Fig. 7. (a) Far-field radiation patterns of anatase $\mathrm{TiO}_{2}$ microsphere array LEDs with various $\mathrm{TiO}_{2}$ sphere diameters. (b) Angular dependent power density comparison of anatase $\mathrm{TiO}_{2}$ microsphere array LEDs. The planar LED is included as reference.

finding will serve as useful guide for optimizing the light extraction in microsphere LEDs deposited by RCD method. Future works will also include the optimization of the microlens array LEDs, which can be formed by embedding the microsphere arrays with polystyrene (PS) materials (i.e., $\mathrm{SiO}_{2} / \mathrm{PS}$ microlens arrays [38]-[41]).

\section{REFERENCES}

[1] M. H. Crawford, "LEDs for solid-state lighting: Performance challenges and recent advances," IEEE J. Sel. Topics Quantum Electron., vol. 15, no. 4, pp. 1028-1040, Jul./Aug. 2009.

[2] Y. K. Ee, J. M. Biser, W. J. Cao, H. M. Chan, R. P. Vinci, and N. Tansu, "Metalorganic vapor phase epitaxy of III-nitride light-emitting diodes on nanopatterned AGOG sapphire substrate by abbreviated growth mode," IEEE J. Sel. Topics Quantum Electron., vol. 15, no. 4, pp. 1066-1072, Jul./Aug. 2009.

[3] N. F. Gardner, G. O. Muller, Y. C. Shen, G. Chen, S. Watanabe, W. Gotz, and M. R. Krames, "Blue-emitting InGaN-GaN double-heterostructure light emitting diodes reaching maximum quantum efficiency above $200 \mathrm{~A} / \mathrm{cm}^{2}$," Appl. Phys. Lett., vol. 91, no. 24, p. 243506, 2007.

[4] H. Zhao, J. Zhang, G. Liu, and N. Tansu, "Surface plasmon dispersion engineering via double-metallic $\mathrm{Au} / \mathrm{Ag}$ layers for III-nitride based light emitting diodes," Appl. Phys. Lett., vol. 98, p. 151115, 2011.

[5] X. Li, S. G. Bishop, and J. J. Coleman, "GaN epitaxial lateral overgrowth and optical characterization," Appl. Phys. Lett., vol. 73, no. 9, pp. 1179-1181, Aug. 1998.

[6] I. H. Brown, P. Blood, P. M. Smowton, J. D. Thomson, S. M. Olaizola, A. M. Fox, P. J. Parbrook, and W. W. Chow, "Time evolution of the screening of piezoelectric fields in InGaN quantum wells," IEEE $J$. Quantum Electron., vol. 42, no. 12, pp. 1202-1208, Nov.-Dec. 2006.

[7] J. H. Ryou, P. D. Yoder, J. Liu, Z. Lochner, H. Kim, S. Choi, H. J. Kim, and R. D. Dupuis, "Control of quantum-confined stark effect in InGaN-based quantum wells," IEEE J. Sel. Topics Quantum Electron., vol. 15, no. 4, pp. 1080-1091, Jul./Aug. 2009. 
[8] X. Li, H. Y. Liu, X. Ni, U. Ozgur, and H. Morkoc, "Effect of carrier spillover and Auger recombination on the efficiency droop in InGaN-based blue LEDs," Superlattices and Microstructures, vol. 47, pp. 118-122, 2010.

[9] B. N. Pantha, I. Feng, K. Aryal, J. Li, J. Y. Lin, and H. X. Jiang, "Erbium-doped AlInGaN alloys as high-temperature thermoelectric materials," Appl. Phys. Express, vol. 4, no. 5, p. 051001, May 2011.

[10] J. Zhang, H. Tong, G. Liu, J. A. Herbsommer, G. S. Huang, and N. Tansu, "Characterizations of seebeck coefficients and thermoelectric figures of merit for AlInN alloys with various in-contents," J. Appl. Phys., vol. 109, no. 5, pp. 053706-1-053706-6, Mar. 2011.

[11] R. M. Farrell, D. F. Feezell, M. C. Schmidt, D. A. Haeger, K. M. Kelchner, K. Iso, H. Yamada, M. Saito, K. Fujito, D. A. Cohen, J. S. Speck, S. P. DenBaars, and S. Nakamura, "Continuous-wave operation of AlGaN-cladding-free nonpolar m-plane $\mathrm{InGaN} / \mathrm{GaN}$ laser diodes," Jpn. J. Appl. Phys., vol. 46, no. 32, pp. L761-L763, Aug. 2007.

[12] M. Kneissl et al., "Continuous-wave operation of ultraviolet InGaN/ InAlGaN multiple-quantum-well laser diodes," Appl. Phys. Lett., vol. 82, no. 15, pp. 2386-2388, Feb. 2003.

[13] R. Dahal, B. Pantha, J. Li, J. Y. Lin, and H. X. Jiang, "InGaN/GaN multiple quantum well solar cells with long operating wavelengths," Appl. Phys. Lett., vol. 94, no. 6, pp. 063505-1-063505-3, Feb. 2009.

[14] A. I. Zhmakin, "Enhancement of light extraction from light emitting diodes," Phys. Rep., vol. 498, pp. 189-241, Feb. 2011.

[15] H. Zhao, G. Y. Liu, J. Zhang, J. D. Poplawsky, V. Dierolf, and N. Tansu, "Approaches for high internal quantum efficiency green InGaN lightemitting diodes with large overlap quantum wells," Opt. Express, vol. 19, no. S4, pp. A991-A1007, Jul. 2011.

[16] R. A. Arif, Y. K. Ee, and N. Tansu, "Polarization engineering via staggered InGaN quantum wells for radiative efficiency enhancement of light emitting diodes," Appl. Phys. Lett., vol. 91, p. 091110, Aug. 2007.

[17] S. H. Park, D. Ahn, and J. W. Kim, "High-efficiency staggered $530 \mathrm{~nm}$ InGaN/InGaN/GaN quantum-well light-emitting diodes," Appl. Phys. Lett., vol. 94, no. 4, pp. 041109-1-041109-3, Jan. 2009.

[18] C. T. Liao, M. C. Tsai, B. T. Liou, S. H. Yen, and Y. K. Kuo, "Improvement in output power of a $460 \mathrm{~nm}$ InGaN light-emitting diode using staggered quantum well," J. Appl. Phys., vol. 108, p. 063107, 2010 .

[19] U. Ozgur, H. Liu, X. Li, and H. Morkoc, "GaN-based light-emitting diodes: Efficiency at high injection levels," Proc. IEEE, vol. 98, no. 7, pp. 1180-1196, 2010

[20] C. C. Kao et al., "Light-output enhancement of nano-roughened GaN laser lift-off light-emitting diodes formed by ICP dry etching," IEEE Photon. Techno. Lett., vol. 19, pp. 849-851, May-Jun. 2007.

[21] C. Huh, K. S. Lee, E. J. Kang, and S. J. Park, "Improved light-output and electrical performance of InGaN-based, light-emitting diode by microroughening of the p-GaN surface," J. Appl. Phys., vol. 93, pp. 9383-9385, Jun. 2003.

[22] T. Fujii, Y. Gao, R. Sharma, E. L. Hu, S. P. DenBaars, and S. Nakamura, "Increase in the extraction efficiency of GaN-based light-emitting diodes via surface roughening," Appl. Phys. Lett., vol. 84, pp. $855-857,2004$

[23] C. F. Lin, Z. H. Yang, J. H. Zheng, and J. H. Dai, "Enhanced light output in nitride-based light-emitting diodes by roughening the mesa sidewall," IEEE Photonic Tech. L., vol. 17, pp. 2038-2040, Oct. 2005.

[24] H. W. Huang et al., "Enhanced light output of an InGaN/GaN light emitting diode with a nano-roughened p-GaN surface," Nanotechnol., vol. 16, pp. 1844-1848, Sept. 2005.

[25] H. W. Choi et al., "GaN micro-light-emitting diode arrays with monolithically integrated sapphire microlenses," Appl. Phys. Lett., vol. 84, pp. 2253-2255, Mar. 2004.

[26] J. S. Lee, J. Lee, S. Kim, and H. Jeon, "Fabrication of reflective GaN mesa sidewalls for the application to high extraction efficiency LEDs," Phys. Status Solid. (c), vol. 4, pp. 2625-2628, 2007.

[27] J. Q. Xi, H. Luo, A. J. Pasquale, J. K. Kim, and E. F. Schubert, "Enhanced light extraction in GaInN light-emitting diode with pyramid reflector," IEEE Photon. Technol. Lett., vol. 18, pp. 2347-2349, Nov. -Dec. 2006.

[28] E. Matioli et al., "High extraction efficiency GaN-based photonic-crystal light-emitting diodes: Comparison of extraction lengths between surface and embedded photonic crystals," Appl. Phys. Express, vol. 3, 2010.

[29] J. J. Wierer, A. David, and M. M. Megens, "III-nitride photonic-crystal light-emitting diodes with high extraction efficiency," Nat. Photon., vol. 3, pp. 163-169, Mar. 2009.
[30] J. Jewell et al., "Double embedded photonic crystals for extraction of guided light in light-emitting diodes," Appl. Phys. Lett., vol. 100, 2012, Art. 171105

[31] C. Wiesmann, K. Bergenek, N. Linder, and U. T. Schwarz, "Photonic crystal LEDs-Designing light extraction," Laser Photon. Rev., vol. 3, pp. 262-286, May 2009.

[32] M. Fujita, S. Takahashi, Y. Tanaka, T. Asano, and S. Noda, "Simultaneous inhibition and redistribution of spontaneous light emission in photonic crystals," Science, vol. 308, pp. 1296-1298, May 27, 2005.

[33] J. Q. Xi et al., "Optical thin-film materials with low refractive index for broadband elimination of Fresnel reflection," Nat. Photon., vol. 1, pp. 176-179, Mar. 2007.

[34] S. Chhajed, W. Lee, J. Cho, E. F. Schubert, and J. K. Kim, "Strong light extraction enhancement in GaInN light-emitting diodes by using self-organized nanoscale patterning of p-type GaN," Appl. Phys. Lett., vol. 98, p. 071102, Feb. 2011.

[35] P. Zhao and H. Zhao, "Analysis of light extraction efficiency enhancement for thin-film-flip-chip InGaN quantum wells light-emitting diodes with GaN micro-domes," Opt. Express, vol. 20, pp. A765-A776, Sep. 2012.

[36] P. Zhao, L. Han, M. R. McGoogan, and H. Zhao, "Analysis of TM mode light extraction efficiency enhancement for deep ultraviolet $\mathrm{AlGaN}$ quantum wells light-emitting diodes with III-nitride micro-domes," Opt. Mater. Express, vol. 2, no. 10, pp. 1397-1406, Oct. 2012

[37] M. Ma et al., "Strong light-extraction enhancement in GaInN lightemitting diodes patterned with $\mathrm{TiO}_{2}$ micro-pillars with tapered sidewalls," Appl. Phys. Lett., vol. 101, p. 141105, 2012.

[38] Y. K. Ee, P. Kumnorkaew, R. A. Arif, J. F. Gilchrist, and N. Tansu, "Enhancement of light extraction efficiency of InGaN quantum wells light emitting diodes using $\mathrm{SiO}_{2}$ /polystyrene microlens arrays," Appl. Phys. Lett., vol. 91, no. 22, pp. 221107-1-221107-3, Nov. 2007.

[39] Y. K. Ee, P. Kumnorkaew, R. A. Arif, H. Tong, H. Zhao, J. F. Gilchrist, and N. Tansu, "Optimization of light extraction efficiency of III-nitride LEDs with self-assembled colloidal-based microlenses," IEEE J. Sel. Topics Quantum Electron., vol. 15, no. 4, pp. 1218-1225, Jul./Aug. 2009.

[40] P. Kumnorkaew, Y. K. Ee, N. Tansu, and J. F. Gilchrist, "Investigation of the deposition of microsphere monolayers for fabrication of microlens arrays," Langmuir, vol. 24, no. 21, pp. 12150-12157, Nov. 2008.

[41] X. H. Li, R. Song, Y. K. Ee, P. Kumnorkaew, J. F. Gilchrist, and N. Tansu, "Light extraction efficiency and radiation patterns of III-nitride light emitting diodes with colloidal microlens arrays with various aspect ratios," IEEE Photon. J., vol. 3, no. 3, pp. 489-499, Jun. 2011.

[42] Y. K. Ee, P. Kumnorkaew, R. A. Arif, H. Tong, J. F. Gilchrist, and N. Tansu, "Light extraction efficiency enhancement of InGaN quantum wells light-emitting diodes with polydimethylsiloxane concave microstructures," Opt. Express, vol. 17, no. 16, pp. 13747-13757, Aug. 2009.

[43] W. H. Koo, W. Youn, P. F. Zhu, X. H. Li, N. Tansu, and F. So, "Light extraction of organic light emitting diodes using defective hexagonalclose-packed array," Adv. Funct. Mater., vol. 22, pp. 3454-3459, Aug. 2012.

Peifen Zhu was born in 1980. She received the B.S. degree in physics from Liaocheng University in 2004, the M.S. degree in physics from Jilin University in 2007, and the M.S. degree in electrical engineering from Texas Tech University in 2011, and is currently working toward the Ph.D. degree in Department of Electrical and Computer Engineering (ECE) and Center for Photonics and Nanoelectronics (CPN), Lehigh University, Bethlehem, PA, USA.

Her research areas are related to III-Nitride semiconductor nanostructures for solid state lighting technologies. Her research works cover the theoretical and experimental aspects of the physics in nano-scale semiconductor, MOCVD and device fabrications of III-Nitride semiconductor devices on $\mathrm{GaN}$ substrates. Her research interests include phosphor-converted white LED, light extraction efficiency of LED, solar hydrogen based on III-V semiconductor.

Ms. Zhu is the recipient of the Dean's Scholarship (Lehigh University). 
Guangyu Liu received the B.S. degree in electronic science and technology from Huazhong University of Science and Technology, China, in 2008, and is currently working toward the Ph.D. degree in Department of Electrical and Computer Engineering (ECE) and Center for Photonics and Nanoelectronics (CPN), Lehigh University, Bethlehem, PA, USA.

Her research interests are related to III-Nitride semiconductor nanostructures and optoelectronics devices, covering the theoretical/computational, metalorganic chemical vapor deposition (MOCVD) growth, and device fabrication and characterization technology. The research topics include the pursuit of high-performance III-Nitride light-emitting diodes (LEDs), the growths of III-Nitride quantum dots (QDs) devices for solid state lighting and solar cells, and investigation of III-Nitride based intersubband quantum cascade laser (QCL). She has published more than 50 refereed journal and conference publications.

Ms. Liu is also a recipient of SPIE Scholarship in Optics and Photonics (Lehigh University), Sherman-Fairchild Fellowship (Lehigh University), the Dean's Scholarship (Lehigh University), and Best Graduation Thesis (Huazhong University of Science and Technology).

Jing Zhang received the B.S. degree in electronic science and technology from Huazhong University of Science and Technology, China, in 2009, and is currently working toward the Ph.D. degree in Department of Electrical and Computer Engineering (ECE) and Center for Optical Technologies (COT) at Lehigh University, Bethlehem, PA, USA.

Her research areas are related to III-Nitride semiconductor nanostructures for thermoelectric and solid state lighting technologies. Her research works cover various aspects of computational, growths, and device fabrication of III-Nitride semiconductor for photonics and thermoelectric applications. Her research topics include the pursuit of novel materials for high thermoelectric figure of merit, and solid state lighting applications.

Ms. Zhang is a recipient of the Dean's Scholarship (2009, Lehigh University), Best Graduation Thesis (2009, Huazhong University of Science and Technology), and SPIE Scholarship in Optics and Photonics (Lehigh University).
Nelson Tansu was born in 1977. He received the B.S. degree in applied mathematics, electrical engineering, and physics (with Highest Distinction) and the $\mathrm{Ph} . \mathrm{D}$. degree in electrical engineering/applied physics from the University of Wisconsin-Madison, Madison, WI, USA, in 1998 and 2003, respectively.

Since July 2003 , he has been a faculty member in the Department of Electrical and Computer Engineering (ECE) and Center for Photonics and Nanoelectronics (CPN) at Lehigh University, Bethlehem, PA, USA, where he currently is the Class of 1961 Associate Professor (with tenure). He currently serves as Associate Editor for OSA Optical Materials Express (2010-present), Assistant/ Associate Editor for Nanoscale Research Letters (2007-present), and Editorin-Chief for Optics (2013-present). He has published in over 240 refereed international journals $(92)$ and conference $(150+)$ publications, and holds several U.S. patents (total > 10). He also regularly reviews leading journals in applied physics, quantum electronics, nanotechnology, photonics, and optoelectronics areas. Previously, he has given numerous lectures, seminars, and keynote and invited talks (total $>45$ ) at universities, research institutions, and conferences in the Canada, Europe, and Asia. His research works cover both the theoretical and experimental aspects of the physics of semiconductor optoelectronics materials and devices, the physics of low-dimensional semiconductor (nanostructure), and MOCVD and device fabrications of III-Nitride and III-V-Nitride semiconductor optoelectronics devices on GaAs, InP, and GaN substrates.

Dr. Tansu served as the Primary Guest Editor of the IEEE JOURNAL OF SELECTED Topics IN QUANTUM ELECTRONICS (2008-2009) and IEEE/OSA Journal of Display TeChNology (2012-2013), and he also serves as an Associate Editor for IEEE PHOTONICS JoURNAL (2009-present). He served on the Technical Program Committee for several major technical conferences for IEEE, OSA, SPIE, and APS, including IEEE/OSA Conference on Lasers and Electro-Optics (2007-2009, 2013), SPIE Photonics West (2009-2013), APS March Annual Meeting (2007, 2009-2011), and ACP (2012, 2013), and others. He was selected as Invited General Participant at the 2008 National Academy of Engineering (NAE)'s U.S. Frontiers of Engineering (FOE) Symposium, and he served as the Organizing Committee for the 2009 NAE's U.S. Frontiers of Engineering Symposium. Recently, he has been invited to participate in the NAE's 2012 German-American Frontiers of Engineering Symposium (GAFOE). 